\title{
Nutritional Status and Intestinal Parasite in School Age Children: A Comparative Cross-Sectional Study
}

\author{
Berhanu Elfu Feleke \\ Department of Epidemiology and Biostatistics, University of Bahir Dar, Bahir Dar, Ethiopia \\ Correspondence should be addressed to Berhanu Elfu Feleke; elfufeleke@gmail.com
}

Received 18 March 2016; Accepted 3 August 2016

Academic Editor: Samuel Menahem

Copyright ( 2016 Berhanu Elfu Feleke. This is an open access article distributed under the Creative Commons Attribution License, which permits unrestricted use, distribution, and reproduction in any medium, provided the original work is properly cited.

\begin{abstract}
Background. The objectives of this study were to determine the burden of underweight and intestinal parasitic infection in the urban and rural elementary school children. Methods. A comparative cross-sectional study design was conducted. Binary logistic regression was used to identify the determinants of malnutrition or intestinal parasites. Two independent samples' $t$-test was used to identify the effect of malnutrition on school performance or hemoglobin level. Results. A total of 2372 students were included. Quarters (24.8\%) of school children were underweight. Underweight was associated with sex [adjusted odds ratio (AOR) 0.61; 95\% $\mathrm{CI}=0.47-0.78]$, age $[\mathrm{AOR}=0.21 ; 95 \% \mathrm{CI}=0.16-0.28]$, intestinal parasitic infection $[\mathrm{AOR} 2.67 ; 95 \% \mathrm{CI}=2-3.55]$, and family size [AOR 23; 95\% CI $=17.67-30.02]$. The prevalence of intestinal parasite among school children was $61.7 \%$ [95\% CI $=60 \%-64 \%]$. Shoe wearing practice [AOR 0.71; 95\% CI $=0.58-0.87$ ], personal hygiene [AOR 0.8; 95\% CI $=0.65-0.99$ ], availability of latrine [AOR $0.34 ; 95 \% \mathrm{CI}=0.27-0.44$ ], age [AOR $0.58 ; 95 \% \mathrm{CI}=0.48-0.7$ ], habit of eating raw vegetables [AOR 3.71; $95 \% \mathrm{CI}=3.01-4.46]$, and family size $[$ AOR $1.96 ; 95 \% \mathrm{CI}=1.57-2.45]$ were the predictors of intestinal parasitic infection.
\end{abstract}

\section{Introduction}

Malnutrition is the cellular imbalance between supply of nutrients, energy, and the body demand for them to ensure growth, maintenance, and specific functions [1]. Underweight is a malnutrition stage in which weight for age of the child is less than $2 \mathrm{SD}$ values (standard deviation) of the WHO (World Health Organization) child growth standards median. It indicates both acute and chronic malnutrition [2]. Globally, more than 870 million people are undernourished and 852 million of them were in developing countries [3]. More than $25 \%$ of children in developing countries were underweight, and $50 \%$ of children deaths were as a result of their poor nutritional status $[4,5]$.

Intestinal parasitic infections were one of the 17 th neglected tropical diseases listed by the World Health Organization. Neglected tropical diseases accounts for the top 4th leading cause of communicable diseases and constituted 46-57 million disability adjusted life years lost $[4,5]$. The common features of neglected tropical diseases are a proxy to poverty and disadvantage, affect populations with low visibility and political voice, cause stigma and discrimination, have an important impact on morbidity and mortality, neglected by researcher, and can be controlled effectively with low cost [6].

Worldwide, approximately more than 2 billion people are infected with helmets infection [4,7-11]. In Africa more than 173 million people are infected with Ascaris lumbricoides, more than 162 million cases of trichiasis, and more than 198 million cases of hook worm [6,12-14]. In developing countries $12 \%$ of the global disease burdens due to intestinal worm were estimated to occur on children 5-14 years old [15].

Intestinal parasitic infections were the leading cause of infection in sub-Saharan Africa resulting in avoidable death. Soil transmitted helmenths infection constitutes $80 \%$ of the neglected tropical disease burden [4, 16-22]. The effect of intestinal parasites was not limited to morbidity and mortality; their impact extends to cognitive impairment, malnutrition, anemia, decreased school attendance, increased susceptibility of the host for infections like HIV/AIDS and tuberculosis, and impairing the growth of the child and adult productivity. They also impose serious socioeconomic impact on the development of a nation $[4,9,11,17,23-28]$.

Less emphasis was given to intestinal parasite prevention and control by decision makers. Intervention against 
intestinal parasitic infection can be implemented with low cost, their treatment cost less than 1 dollar per case $[4,11]$. Intervention against the parasitic disease was among the cost effective intervention; for every dollar spent on deworming the society will gain more than 30 dollars [29].

Nutritional status and intestinal parasitic infections were assessed only for children less than five years of age. There are no data that can show the nutritional status and intestinal parasitic infection of children above 5 years of age, so this study was conducted to fill these gaps. The effects of nutritional status or intestinal parasite were mainly studied on the morbidity and mortality of the children and this study will increase the intersectoral collaboration of decision makers in the area of health and education by showing the effect of nutritional status or intestinal parasite on the school performance of children. Usually both intestinal parasitic infections and malnutrition were studied in rural setting and their burden on the urban areas was not properly assessed, so this study will answer the question that malnutrition or intestinal parasites were the problem of urban area or not.

\section{Objectives}

The objectives of this study were

(i) to describe the burden of malnutrition among school children in urban and rural setting;

(ii) to describe the burden of intestinal parasitic infection among school children in urban and rural setting;

(iii) to identify the determinants of malnutrition and intestinal parasitic infection in urban and rural setting;

(iv) to identify the effect of malnutrition and intestinal parasitic infection on the school performance of children.

\section{Methods}

A comparative cross-sectional study design was implemented in urban and rural settings. The study was conducted in the city of Bahir Dar (urban setting) and Mecha District (rural setting). Bahir Dar City was located 578 kilometers northwest of Addis Ababa (the capital city of Ethiopia). Bahir Dar City contains 37 elementary schools with a total of 45,740 students. Also the city contains 10 governmental health centers, one government hospital, one private hospital, and more than 20 private clinics. Mecha District is a rural area located $40 \mathrm{~km}$ to the north of Bahir Dar with 40 kebeles (the smallest administrative unit in Ethiopia). The district contains 101 elementary schools and 80,727 children in these elementary schools. The district contains 10 governmental health centers and 26 health posts.

The target population was all elementary school age children. The study population was selected elementary school students from Bahir Dar City and Mecha District. Students that received anthelmentic medication in the past 4 weeks or students unable to give stool and blood samples were excluded. The sample size was calculated using Epiinfo software version 7 assuming 95\% CI, 90\% power, 50\% malnutrition or intestinal parasitic infection in rural children, odds ratio of 1.5 , design effect of 1.8 , a rural to an urban ratio of 2 , and a $15 \%$ nonresponse rate. The final sample size was 2,509 students. A multistage sampling technique was used to select the school children. For Bahir Dar, first 10 elementary schools were selected from a total of 37 elementary schools using the lottery method. Then, simple random sampling technique was used to select children from the 10 schools. For Mecha District, first 9 kebeles were selected from a total of 40 cables using the lottery method, one elementary school was selected from each kebele by simple random sampling using lottery method, and finally the required number of children were selected from the 9 schools by simple random sampling technique. The data were collected from March to May, 2014.

The data collection process contains 3 parts: interviewing the parents/guardians of the children, measuring anthropometric indicators, and collecting the stool and blood samples from the children.

For the interview part first the questioner was prepared in English then translated to Amharic (local language) then back to English to keep its consistency. The interview was conducted by 25 diploma nurse professionals and supervised by 5 degree holder health professionals. School performance was measured by using their previous semester CGPA (cumulative grade point average) of students.

3.1. Anthropometry. Weight and height of each child were measured by a clinical nurse. Digital weight scale was used to measure the weight of each child and weight was measured to the nearest 0.1 kilograms. Height was measured using the vertical measuring rod to the nearest $0.1 \mathrm{cms}$. The child's shoes, hair clips, and braids were removed before measurement, children were positioned feet together flat on the ground, heels touching the back plate of the measuring instrument, legs straight, buttocks against the backboard, scapula against the backboard, and arms were loosely at their side.

3.2. Blood and Stool Samples. The blood and stool samples were collected by 8 first degree holder laboratory technologists and supervised by 2 second degree holder laboratory technologists. From each student one gram stool sample was collected in $10 \mathrm{~mL}$ SAF (Sodium Acetate-Acetic AcidFormalin) solution. A concentration technique was used. The stool sample was well mixed and filtered using a funnel with gauze and then centrifuged for one minute at 2000 RPM (Revolution Per Minute) and the supernatant was discarded. $7 \mathrm{ML}$ (Milliliter) normal saline was added and mixed with a wooden stick, $3 \mathrm{ML}$ ether was added and mixed well then centrifuged for 5 minutes at 2000 RPM. Finally the supernatant was discarded and the whole sediment was examined for parasites [30]. $1 \mathrm{ML}$ blood sample was collected from each child following standard operational procedures to measure the hemoglobin level of children using Mindray hematology analyzer.

To maintain the quality of the data pretest was conducted with 50 parents, training was given for data collectors and 
supervisors and the whole data collection process were closely supervised by the investigator and supervisors. The collected data were checked for its completeness. The data were entered in the computer using Epi-info software by 10 IT (Information Technology) technicians and analyzed using SPSS software version 20 and WHO Anthro-plus software.

To identify the prevalence of intestinal parasites or nutritional status of the children descriptive statistics were used. Binary logistic regression was used to identify the determinants of malnutrition or intestinal parasites. Variables with $p$ value less than 0.05 and $95 \%$ CI were used to discover the determinants of nutritional status or intestinal parasite. Two independent samples' $t$-test was used to identify the effect of malnutrition on school performance or hemoglobin level.

\section{Ethics Statement}

Ethical clearance was granted from Amhara National Regional State Health Bureau ethical committee. Permission was obtained from the school directors; written informed consent was obtained from each study's participants; for minors, written informed consent was obtained from parents or guardians of the children. The confidentiality of the data was kept at all steps. Study participants right to withdraw from the study at any point was respected. Students with intestinal parasites or low hemoglobin counts were referred to the nearby health center for further management.

\section{Results}

5.1. Population Profile. A total of 2372 students was included giving a response rate of $94.54 \%$. The mean age of the students was 12.75 years (standard deviation $(\mathrm{SD})=2.92$ years). Males constituted $51.4 \%$ (1220) of the study participants. The mean height of the students was 151.2 centimeters ( $\mathrm{SD}=16.17 \mathrm{CM})$. The mean weight of the students was $41.32 \mathrm{KG}(\mathrm{SD}=14.1 \mathrm{KG})$ (Table 1).

\section{Malnutrition (Underweight)}

A quarter of school children $(24.8 \%, 95 \%$ CI $[23 \%-26 \%])$ were malnourished and $10.8 \%$ of school children were severely malnourished. After adjusting for age, sex, intestinal parasite infection, residence, mother education, average monthly income, availability of latrine, parent work, and family size, malnutrition was associated with sex of the child, age, intestinal parasitic infection, and family size (Table 2 ).

The prevalence of underweight in female school age children was $21.96 \%$ and the prevalence of underweight in male age children was $27.13 \%$. This result was statistically significant ( $p$ value less than 0.01). Being of female gender decreases the risk of underweight by $39 \%$ [AOR $0.61 ; 95 \%$ $\mathrm{CI}=0.47-0.78]$. Availability of latrine decreases the risk of underweight by $36 \%$ [AOR $0.64 ; 95 \% \mathrm{CI}=0.48-0.85$ ]. The prevalence of underweight in school children whose age was greater than or equal to 13 years was $11.67 \%$, whereas prevalence of underweight in children whose age was less than
TABLE 1: Population profile of the study participants $(n=2372)$.

\begin{tabular}{|c|c|c|c|}
\hline $\mathrm{SN}$ & Population profile & Frequency & Percentage \\
\hline \multirow{4}{*}{ (1) } & Age (in years) & & \\
\hline & $5-9$ & 384 & 16.2 \\
\hline & $10-14$ & 1296 & 54.6 \\
\hline & $15-19$ & 692 & 29.2 \\
\hline \multirow{3}{*}{ (2) } & Sex & & \\
\hline & Male & 1220 & 51.4 \\
\hline & Female & 1152 & 48.6 \\
\hline \multirow{6}{*}{ (3) } & Height (in CM) & & \\
\hline & $103-122$ & 130 & 5.5 \\
\hline & $123-142$ & 511 & 21.5 \\
\hline & $143-162$ & 1021 & 43 \\
\hline & $163-182$ & 660 & 27.8 \\
\hline & $>182$ & 50 & 2.1 \\
\hline \multirow{5}{*}{ (4) } & Weight (in KG) & & \\
\hline & $15-34$ & 963 & 40.6 \\
\hline & $35-54$ & 942 & 39.7 \\
\hline & $55-74$ & 460 & 19.4 \\
\hline & $75-94$ & 7 & 0.3 \\
\hline \multirow{3}{*}{ (5) } & Grade & & \\
\hline & $0-4$ & 423 & 17.8 \\
\hline & $5-8$ & 1949 & 82.2 \\
\hline \multirow{3}{*}{ (6) } & Residence & & \\
\hline & Rural & 1578 & 66.5 \\
\hline & Urban & 794 & 33.5 \\
\hline \multirow{3}{*}{ (7) } & Source of water & & \\
\hline & Pipe & 1459 & 61.5 \\
\hline & Other & 913 & 38.5 \\
\hline \multirow{3}{*}{ (8) } & Income per month & & \\
\hline & $<1000$ birrs & 1598 & 67.4 \\
\hline & $\geq 1000$ birrs & 774 & 32.6 \\
\hline \multirow{3}{*}{ (9) } & Availability of latrine & & \\
\hline & Available & 1805 & 76.1 \\
\hline & Not available & 567 & 23.9 \\
\hline \multirow{3}{*}{ (10) } & Work of parent & & \\
\hline & Farmer & 1344 & 56.7 \\
\hline & Other & 1028 & 43.3 \\
\hline
\end{tabular}

13 years was $33.24 \%$. This result was statistically significant ( $p$ value $<0.01$ ). School children whose age is greater than or equal to 13 years were $79 \%$ protected from being underweight as compared to children whose age was less than 13 years [AOR $0.21 ; 95 \% \mathrm{CI}=0.16-0.28$ ]. The odds of underweight in school children with intestinal parasitic infection increased 2.67-fold as compared to children with no intestinal parasitic infection $[95 \% \mathrm{CI}=2.00-3.55]$. Family size directly affects the nutritional status of children; the odds of underweight in school children with family size greater than 4 were 23 -fold higher as compared to children with family size less than or equal to 4 [ $95 \% \mathrm{CI}=17.67-30.02]$. The burden of malnutrition on urban and rural school children was the same. 
TABLE 2: Predictors of underweight in school age children $(n=2372)$.

\begin{tabular}{|c|c|c|c|c|c|}
\hline \multirow{2}{*}{ Variable } & \multicolumn{2}{|c|}{ Underweight } & \multirow{2}{*}{ COR $(95 \% \mathrm{CI})$} & \multirow{2}{*}{$\mathrm{AOR}(95 \% \mathrm{CI})$} & \multirow{2}{*}{$p$ value } \\
\hline & Yes & No & & & \\
\hline Sex & & & $0.79[0.62-0.92]$ & $0.61[0.47-0.78]$ & $<0.01$ \\
\hline Female & 253 & 899 & & & \\
\hline Male & 331 & 889 & & & \\
\hline Latrine & & & $0.61[0.5-0.76]$ & $0.64[0.48-0.85]$ & $<0.01$ \\
\hline Available & 403 & 1402 & & & \\
\hline Not available & 181 & 386 & & & \\
\hline Age & & & $0.26[0.2-0.32]$ & $0.21[0.16-0.28]$ & $<0.01$ \\
\hline$\geq 13$ & 116 & 878 & & & \\
\hline$<13$ & 468 & 910 & & & \\
\hline Intestinal parasitic infection & & & $3.34[2.65-4.22]$ & $2.67[2-3.55]$ & $<0.01$ \\
\hline Yes & 471 & 992 & & & \\
\hline No & 113 & 796 & & & \\
\hline Family size & & & 19 [14.98-24.11] & $23[17.67-30.02]$ & $<0.01$ \\
\hline$>4$ & 407 & 193 & & & \\
\hline$\leq 4$ & 177 & 1595 & & & \\
\hline
\end{tabular}

TABLE 3: The determinants of intestinal parasite in school age children.

\begin{tabular}{|c|c|c|c|c|c|}
\hline \multirow{2}{*}{ Variables } & \multicolumn{2}{|c|}{ Intestinal parasite } & \multirow{2}{*}{ COR $[95 \% \mathrm{CI}]$} & \multirow{2}{*}{$\mathrm{AOR}[95 \% \mathrm{CI}]$} & \multirow{2}{*}{$p$ value } \\
\hline & Yes & No & & & \\
\hline Shoe wearing practice & & & $0.52[0.44-0.62]$ & $0.71[0.58-0.87]$ & $<0.01$ \\
\hline Yes & 560 & 493 & & & \\
\hline No & 903 & 416 & & & \\
\hline Personal hygiene & & & $0.55[0.46-0.65]$ & $0.8[0.65-0.99]$ & 0.04 \\
\hline Kept & 517 & 455 & & & \\
\hline Not kept & 946 & 454 & & & \\
\hline Availability of latrine & & & $0.3[0.24-0.38]$ & $0.34[0.27-0.44]$ & $<0.01$ \\
\hline Yes & 1005 & 800 & & & \\
\hline No & 458 & 109 & & & \\
\hline Ingesting raw vegetables & & & $3.8[3.18-4.54]$ & $3.71[3.01-4.46]$ & $<0.01$ \\
\hline Yes & 1044 & 360 & & & \\
\hline No & 419 & 549 & & & \\
\hline Age & & & $0.53[0.44-0.62]$ & $0.58[0.48-0.7]$ & $<0.01$ \\
\hline$\geq 13$ & 525 & 469 & & & \\
\hline$<13$ & 938 & 440 & & & \\
\hline Family size & & & $2.06[1.67-2.54]$ & $1.96[1.57-2.45]$ & $<0.01$ \\
\hline$>4$ & 442 & 158 & & & \\
\hline$\leq 4$ & 1021 & 751 & & & \\
\hline
\end{tabular}

6.1. Intestinal Parasitosis. The prevalence of intestinal parasites was $61.7 \%(95 \% \mathrm{CI}=60 \%-64 \%)$. Hookworm was the predominant intestinal parasite followed by Ascaris lumbricoides. Mixed infections were found in $3.4 \%$ of school children.

After adjusting for age, sex, residence, mother's education, source of water, average monthly income, parent's work, shoes wearing practice, hand washing practices, personal hygiene, availability of latrine, swimming habits, habit of eating raw vegetables, and family size, it is found that shoe wearing practice, personal hygiene, availability of latrine, age, habit of eating raw vegetables, and family size were the predictors of intestinal parasitic infection (Table 3 ).

The odds of intestinal parasitic infection's for children that did not wear shoe increased by $29 \%$ [AOR 0.71; 95\% CI 0.58-0.87]. The prevalence of intestinal parasitic infections in school age children that keep their personal hygiene was $53.19 \%$, the prevalence of intestinal parasitic infections in school age children that did not keep their personal hygiene was $67.57 \%$. This result was statistically significant [AOR 0.8 ; $95 \% \mathrm{CI}=0.65-0.99]$. Availability of latrine decreased the 
TABLE 4: School performance and malnutrition.

\begin{tabular}{lcccc}
\hline Group & Average of previous semester & Mean difference [95\% CI] & $t$ & Degree of freedom \\
\hline Not undernourished & 69.53 & $1.32[0.05-2.28]$ & 2 & 2370 \\
Undernourished & 68.22 & & & $0.04^{*}$ \\
\hline
\end{tabular}

${ }^{*}$ Equal variances assumed.

TABLE 5: Hemoglobin count and malnutrition.

\begin{tabular}{|c|c|c|c|c|c|}
\hline Group & Average hemoglobin count & Mean difference $[95 \% \mathrm{CI}]$ & $t$ & Degree of freedom & $p$ value \\
\hline Not undernourished & 11.65 & \multirow{2}{*}{$0.1[0.03-0.16]$} & \multirow{2}{*}{2.78} & \multirow{2}{*}{2370} & \multirow{2}{*}{$<0.01^{*}$} \\
\hline Undernourished & 11.56 & & & & \\
\hline
\end{tabular}

${ }^{*}$ Equal variances assumed.

risk of intestinal parasitic infections by $66 \%$ [AOR, 0.34: $95 \%$ $\mathrm{CI}=0.27-0.44]$. The odds of intestinal parasitic infections among school children that had a habit of ingesting raw vegetables increased 3.71-fold [ $95 \% \mathrm{CI}=3.01-4.46$ ]. The risk of intestinal parasitic infections decreased by $42 \%$ for children whose age were greater than or equal to 13 years [AOR $0.58 ; 95 \%=0.48-0.7]$. High family size increases the risk of intestinal parasitic infection, the odds of intestinal parasitic infection for high family sized were 2-fold higher than children with low family size [AOR 1.96 ; 95\% CI $=1.57-2.45$ ].

6.2. Effect of Malnutrition. Two independent samples' $t$-test verified that malnutrition directly affects the school performance of children (Table 4).

A two independent samples' $t$-test verified that malnutrition decreased the hemoglobin count of school children (Table 5).

\section{Discussion}

Underweight is common in school children with a prevalence of $24.8 \%$ (95\% CI [23\%-26\%]). This result agrees with findings from northern part of Ethiopia [31-39], higher than finding from west Ethiopia [40]. This is due to improper implementation of effective community health intervention.

The odds of underweight in females were 39\% lower than males [AOR 0.61; 95\% CI $=0.47-0.78$ ]. This result agrees with finding from different scholars [32, 39, 41-43]. This is due to different physiological demand of nutrition for these genders. This might also be due to the reason of the different distribution of morbidity across male gender.

Availability of latrine decreases the risk of underweight by $36 \%$ [AOR 0.64; 95\% CI $=0.48-0.85$ ]. This is due to the fact that latrine utilization prevents the occurrence of intestinal parasite and decreases the occurrence of morbidity associated with intestinal parasite. Also the availability of latrine acts as a signal for the proper implementation of community health preventive activities.

The odds of underweight in school children with intestinal parasitic infection increased 2.67-fold as compared to children with no intestinal parasitic infection $[95 \% \mathrm{CI}=2.00-$ 3.55]. This finding is different from findings from different parts of the world $[17,18,31]$. This is due to the reason that intestinal parasitic infection competes for the nutritional intake of children and intestinal parasitic infection also impairs the immune system of the host so that it makes them susceptible to many diseases [25].

The odds of underweight in school children with large family size were 23-fold higher as compared to children with small family size less [95\% CI $=17.67-30.02]$. This finding agrees with finding from Gondar [32]. This is due to the reason that large family size will share the limited available nutrients inside that house so that children will not get the required nutritional demand for their growth.

The burden of malnutrition was the same on urban and rural elementary school children. Effective community health intervention should be implemented in urban and rural setting.

The prevalence of intestinal parasite was $61.7 \%$ (95\% CI = $60 \%-64 \%)$. This magnitude was lower when compared to finding from Malaysia and Philippines [44, 45] and higher than finding from Cameron and eastern part of Ethiopia $[40,46]$. This might be due to the different implementation strategy of primary healthcare components in these areas.

Personal hygiene greatly reduces the burden of intestinal parasites. The prevalence of intestinal parasitic infection in school age children that keep their personal hygiene was $53.19 \%$, and the prevalence of intestinal parasitic infection in school age children that did not keep their personal hygiene was $67.57 \%$. This result was statistically significant [AOR 0.8 ; $95 \% \mathrm{CI}=0.65-0.99]$. This finding agrees with finding from different parts of the world [47]. This is due to the reason that proper personal hygiene breaks the chain of intestinal parasite transmission.

The odds of intestinal parasitic infection in the presence of latrine decreases by $66 \%$ [AOR $0.34 ; 95 \% \mathrm{CI}=0.27-0.44$ ]. This finding agrees with finding from different parts of the world [32]. This is due to the fact that latrine will dump all the intestinal parasites away from the susceptible host.

The odds of intestinal parasitic infection among school children that had a habit of ingesting raw vegetables increased 3.71-fold [95\% CI $=3.01-4.46]$. This finding agrees with finding from Delgi [48]. This is due to the fact that raw vegetables are good culture media for intestinal parasites. 
The burden of intestinal parasitic infection was higher in early age. The risk of intestinal parasitic infection decreased by $42 \%$ for children whose age is greater than or equal to 13 years [AOR 0.58; 95\% $=0.48-0.7$ ]. This finding agrees with finding from different parts of the world [4]. This is due to the reason that as the age of children increases, they can protect themselves from intestinal parasitic infection because they are active.

The odds of intestinal parasitic infection for high family size children were 2-fold higher than children with low family size [AOR $1.96 ; 95 \% \mathrm{CI}=1.57-2.45]$. This is due to the reason that overcrowding decreases the probability of latrine utilization and personal and environmental hygiene will not be kept if the size of family is large.

The burden of intestinal parasitic infection on urban and rural elementary school students was the same, calling for proper implementation of primary healthcare components in urban and rural areas.

Malnutrition decreases the school performance of children by $1.32 \%$. Improving the nutritional status of school children increases the quality of education. This finding agrees with finding from different parts of the world $[4,8$, 9]. This is due to the reason that proper nutritional intake increases the brain cell development and improves the cognitive performance of the child. Malnutrition also decreases the hemoglobin level of school children by $0.1 \mathrm{mg} / \mathrm{dL}$ making the school children anemic. This is due to the reason that malnourished children will not take enough quantity of iron per the demand of the child.

\section{Conclusions}

Malnutrition (underweight) was higher in urban and rural elementary school students. The nutritional status of children was affected by gender, intestinal parasitic infection, age of the child, family size, and latrine utilization. High prevalence of intestinal parasitic infection was observed in urban and rural elementary school students.

\section{Additional Points}

Recommendation. High level decision makers in the area of health should give special emphasis to the nutritional intervention of the child and on averting the risk of intestinal parasitic infection. Decision makers in the area of child education should include nutritional intervention as a mechanism to increase the quality of child education. Institutions that work in the area of malnutrition should include family planning as one form of keeping the child well nourished.

\section{Disclosure}

The funder has no role in the design, analysis, interpretation of data and in preparation of the manuscript.

\section{Competing Interests}

The author declares that he has no competing interests.

\section{Acknowledgments}

The author would like to acknowledge Federal Democratic Republic of Ethiopia Ministry of Health, for financially sponsoring this paper. He would also like to acknowledge Amhara National Regional Health Bureau and Education Bureau for their unreserved support during the field work. Last but not least he would like to extend his heartfelt appreciation to the school directors and families of the school children for their very nice cooperation during the data collection period.

\section{References}

[1] World Health Organization and UNICEF, "WHO, UNICEF, and SCN informal consultation on community-based management of severe malnutrition in children," SCN Nutrition Policy Paper 21, 2006, http://www.who.int/child_adolescent_ health/documents/fnb_v27n3_suppl/en/index.html.

[2] WHO, Nutritional Landscape Information System, World Health Organization, Geneva, Switzerland, 2010.

[3] Food and Agriculture Organization of the United Nations (FAO), The State of Food Insecirity in the World, Food and Agriculture Organization of the United Nations (FAO), Rome, Italy, 2012.

[4] N. Jeremiah, A. Carol, S. Yulya, and M. Kacie, Social and Economic Impact Review on Neglected Tropical Diseases, Hudson Institute, Washington, DC, USA, 2012.

[5] P. J. Hotez, D. H. Molyneux, A. Fenwick, E. Ottesen, S. Ehrlich Sachs, and J. D. Sachs, "Incorporating a rapid-impact package for neglected tropical diseases with programs for HIV/AIDS, tuberculosis, and malaria," PLoS Medicine, vol. 3, no. 5, article e102, 2006.

[6] WHO, Working to Overcome the Global Impact of Neglected Tropical Diseases, World Health Organization, Geneva, Switzerland, 2010.

[7] L. Savioli, S. Stansfield, D. A. Bundy et al., "Schistosomiasis and soil-transmitted helminth infections: forging control efforts," Transactions of the Royal Society of Tropical Medicine and Hygiene, vol. 96, no. 6, pp. 577-579, 2002.

[8] P. J. Hotez, A. Fenwick, L. Savioli, and D. H. Molyneux, "Rescuing the 'bottom billion' through control of neglected tropical diseases," The Lancet, vol. 373, no. 9674, pp. 1570-1575, 2009.

[9] J. Bethony, S. Brooker, M. Albonico et al., "Soil-transmitted helminth infections: ascariasis, trichuriasis, and hookworm," The Lancet, vol. 367, no. 9521, pp. 1521-1532, 2006.

[10] C. M. Noyer and L. J. Brandt, "Parasitic infections of the gastrointestinal tract," Current Gastroenterology Reports, vol. 1, no. 4, pp. 282-291, 1999.

[11] P. J. Hotez, A. Fenwick, L. Savioli, and D. H. Molyneux, "Rescuing the bottom billion through control of neglected tropical diseases," The Lancet, vol. 373, no. 9674, pp. 1570-1575, 2009.

[12] D. H. Molyneux, P. J. Hotez, and A. Fenwick, "'Rapid-impact interventions': how a policy of integrated control for Africa's neglected tropical diseases could benefit the poor," PLoS Medicine, vol. 2, no. 11, article no. e336, 2005.

[13] S. Brooker, A. C. A. Clements, and D. A. P. Bundy, "Global epidemiology, ecology and control of soil-transmitted helminth infections," Advances in Parasitology, vol. 62, pp. 221-261, 2006.

[14] S. Brooker, A. C. A. Clements, P. J. Hotez et al., "The co-distribution of Plasmodium falciparum and hookworm among African schoolchildren," Malaria Journal, vol. 5, article 99, 2006. 
[15] S. Awasthi, D. A. P. Bundy, and L. Savioli, "Helminthic infections," The British Medical Journal, vol. 327, no. 7412, pp. 431-433, 2003.

[16] C. Nokes, E. S. Cooper, B. A. Robinson, and D. A. P. Bundy, "Geohelminth infection and academic assessment in Jamaican children," Transactions of the Royal Society of Tropical Medicine and Hygiene, vol. 85, no. 2, pp. 272-273, 1991.

[17] C. Nokes and D. A. P. Bundy, "Does helminth infection affect mental processing and educational achievement?" Parasitology Today, vol. 10, no. 1, pp. 14-18, 1994.

[18] C. Nokes, S. Grantham, A. Sawyer, E. Cooper, and D. Bundy, "Parasitic helminth infection and cognitive function in schoolchildren," Proceedings of the Royal Society of London B, vol. 247, pp. 77-81, 1992.

[19] L. Stephenson, M. Latham, K. Kurz, S. Kinoti, and H. Brigham, "Treatment with a single dose of albendazole improves growth of Kenyan school children with hookworm, Trichuris trichiura, and Ascaris lumbericoides infections," The American Journal of Tropical Medicine and Hygiene, vol. 41, no. 1, pp. 78-87, 1989.

[20] M.-S. Chan, "The global burden of intestinal nematode infections-fifty years on," Parasitology Today, vol. 13, no. 11, pp. 438443, 1997.

[21] WHO, Basic Laboratory Methods in Medical Parasitology, vol. 32, WHO, Geneva, Switzerland, 1991.

[22] WHO: World Health Organization, "Prevention and control of schistosomiasis and soil-transmitted helminthiasis," WHO Technical Report Series 912, WHO, Geneva, Switzerland, 2002.

[23] E. Daniel, M. Getahun, A. Hannah, and B. Sven, "Are intestinal helminths risk factors for developing active tuberculosis?" Tropical Medicine and International Health, vol. 2, no. 4, pp. 551558, 2006.

[24] L. S. Stephenson, "Optimising the benefits of anthelmintic treatment in children," Paediatric Drugs, vol. 3, no. 7, pp. 495-508, 2001.

[25] C. Nokes and D. A. P. Bundy, "Compliance and absenteeism in school children: implications for helminth control," Transactions of the Royal Society of Tropical Medicine and Hygiene, vol. 87, no. 2, pp. 148-152, 1993.

[26] P. Hadidjaja, E. Bonang, M. A. Suyardi, S. A. N. Abidin, I. S. Ismid, and S. S. Margono, "The effect of intervention methods on nutritional status and cognitive function of primary school children infected with Ascaris lumbricoides," The American Journal of Tropical Medicine and Hygiene, vol. 59, no. 5, pp. 791795, 1998.

[27] C. Nokes, S. M. Grantham-McGregor, A. W. Sawyer, E. S. Cooper, and D. A. P. Bundy, "Parasitic helminth infection and cognitive function in school children," Proceedings of the Royal Society B: Biological Sciences, vol. 247, no. 1319, pp. 77-81, 1992.

[28] C. N. Nyaruhucha, P. S. Mamiro, A. J. Kerengi, and N. B. Shayo, "Nutritional status of underfive children in a pastoral community in Simanjiro district, Tanzania," Tanzania Health Research Bulletin, vol. 8, no. 1, pp. 32-36, 2006.

[29] M. Kremer, The Wisest Investment We Can Make: Using Schools to Fight Neglected Tropical Diseases, 2008.

[30] S. T. Institute, "Methods in parasitology," in Sodium AcetateAcetic Acid-Formalin Solution Method for Stool Specimen, pp. 118, Swiss Tropical Institute, Swiss TPH, Basel, Switzerland, 2005.

[31] B. Amare, J. Ali, B. Moges et al., "Nutritional status, intestinal parasite infection and allergy among school children in Northwest Ethiopia," BMC Pediatrics, vol. 13, article 7, pp. 1-9, 2013.

[32] T. Gultie, E. Sisay, and G. Sebsibie, "Nutritional status and associated factors among orphan children below the age of five years in Gondar City, Ethiopia," Journal of Food and Nutrition Sciences, vol. 2, no. 4, pp. 179-184, 2014.

[33] N. Nam, G. Bizu, A. Nigusu, K. Abera, W. Michelle, and B. Yemane, "Intestinal parasitic infection and nutritional status among school children in Angolela, Ethiopia," Journal of Preventive Medicine and Hygiene, vol. 53, no. 3, pp. 157-164, 2012.

[34] B. Amare, B. Moges, B. Fantahun et al., "Micronutrient levels and nutritional status of school children living in Northwest Ethiopia," Nutrition Journal, vol. 11, article 108, 2012.

[35] B. Abera, G. Alem, M. Yimer, and Z. Herrador, "Epidemiology of soil-transmitted helminths, Schistosoma mansoni, and haematocrit values among schoolchildren in Ethiopia," Journal of Infection in Developing Countries, vol. 7, no. 3, pp. 253-260, 2013.

[36] A. Amha, A. Ahmed, and K. Abera, "Assessment of demographic, health and nutrition related factors to a school performance among school children in Arb-Gebeya Town, TachGaynt Woreda, South Gondar, Ethiopia," Ethiopian Journal of Health Development, vol. 27, no. 2, pp. 104-110, 2013.

[37] M. A. Mahmud, M. Spigt, A. Mulugeta Bezabih, I. López Pavon, G.-J. Dinant, and R. Blanco Velasco, "Risk factors for intestinal parasitosis, anaemia, and malnutrition among school children in Ethiopia," Pathogens and Global Health, vol. 107, no. 2, pp. $58-$ 65, 2013.

[38] B. Mathewos, A. Alemu, D. Woldeyohannes et al., "Current status of soil transmitted helminths and Schistosoma mansoni infection among children in two primary schools in North Gondar, Northwest Ethiopia: a cross sectional study," BMC Research Notes, vol. 7, article 88, 2014.

[39] CSA, CSA: Ethiopia Demographic and Health Survey, CSA, Addia Ababa, Ethiopia, 2011.

[40] G. Tadesse, "The prevalence of intestinal helminthic infections and associated risk factors among school children in Babile town, eastern Ethiopia," Ethiopian Journal of Health Development, vol. 19, no. 2, pp. 140-147, 2005.

[41] CSA, "Ethiopia mini demographic and health survey," in Nutrition of Children and Adults Volume 1, pp. 53-55, Ethiopia Minstry of Health, Addis Ababa, Ethiopia, 2014.

[42] M. Alemu, T. Bekele, W. Tassew et al., Child Nutritional Status in Poor Ethiopian Households, vol. 1, Edited by C. Solomon, Young Lives, Addis Ababa, Ethiopia, 2005.

[43] Z. Mekonnen, S. Meka, A. Zeynudin, and S. Suleman, "Schistosoma mansoni infection and undernutrition among school age children in Fincha'a sugar estate, rural part of West Ethiopia," BMC Research Notes, vol. 7, article 763, 2014.

[44] A. Ahmed, H. M. Al-Mekhlafi, M. N. Azam et al., "Soiltransmitted helminthiasis: a critical but neglected factor influencing school participation of Aboriginal children in rural Malaysia," Parasitology, vol. 139, no. 6, pp. 802-808, 2012.

[45] V. Janice, B. Vicente, and C. Florencia, "Determination of soil-transmitted helminth infection and its association with hemoglobin levels among Aeta schoolchildren of Katutubo Village in Planas, Porac, Pampanga," Philippine Science Letters, vol. 7, no. 1, pp. 73-80, 2014.

[46] J. V. Mbuh and N. E. Nembu, "Malnutrition and intestinal helminth infections in schoolchildren from Dibanda, Cameroon," Journal of Helminthology, vol. 87, no. 1, pp. 46-51, 2013.

[47] K. Ziegelbauer, B. Speich, D. Mäusezahl, R. Bos, J. Keiser, and J. Utzinger, "Effect of sanitation on soil-transmitted helminth infection: systematic review and meta-analysis," PLoS Medicine, vol. 9, no. 1, Article ID e1001162, 2012. 
[48] A. Asrat, D. Tewodros, and W. Alemayehu, "Prevalence and risk factors of intestinal parasites among Delgi school children, North Gondar, Ethiopia," Journal of Parasitology and Vector Biology, vol. 3, no. 5, pp. 75-81, 2011. 


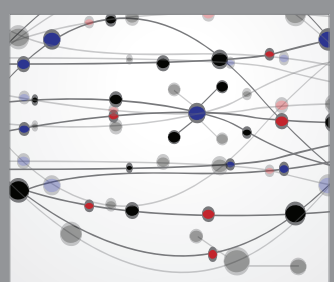

The Scientific World Journal
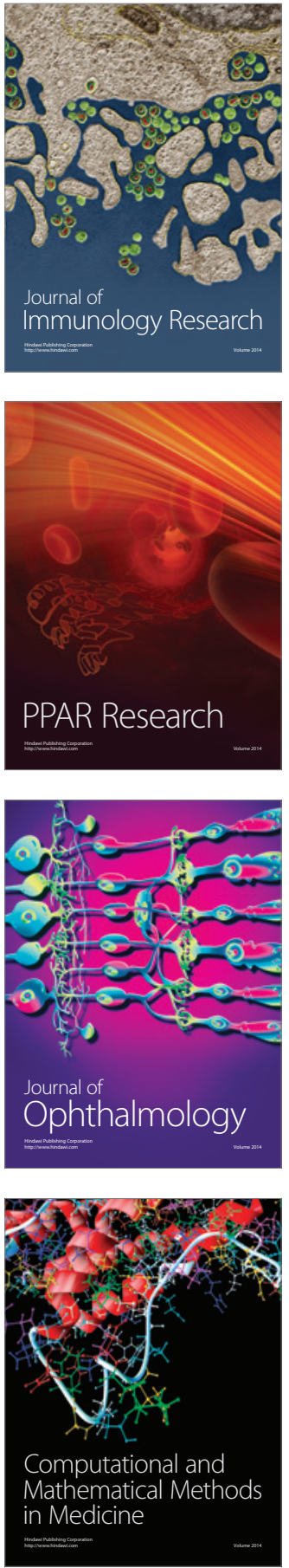

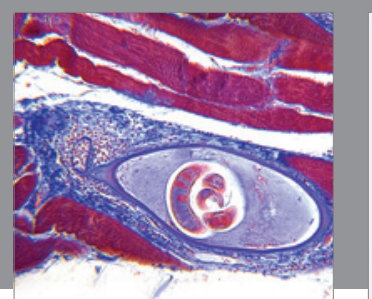

Gastroenterology Research and Practice

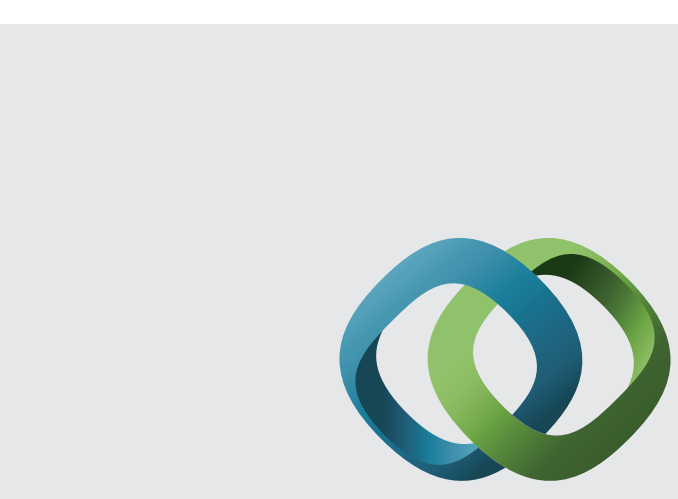

\section{Hindawi}

Submit your manuscripts at

http://www.hindawi.com
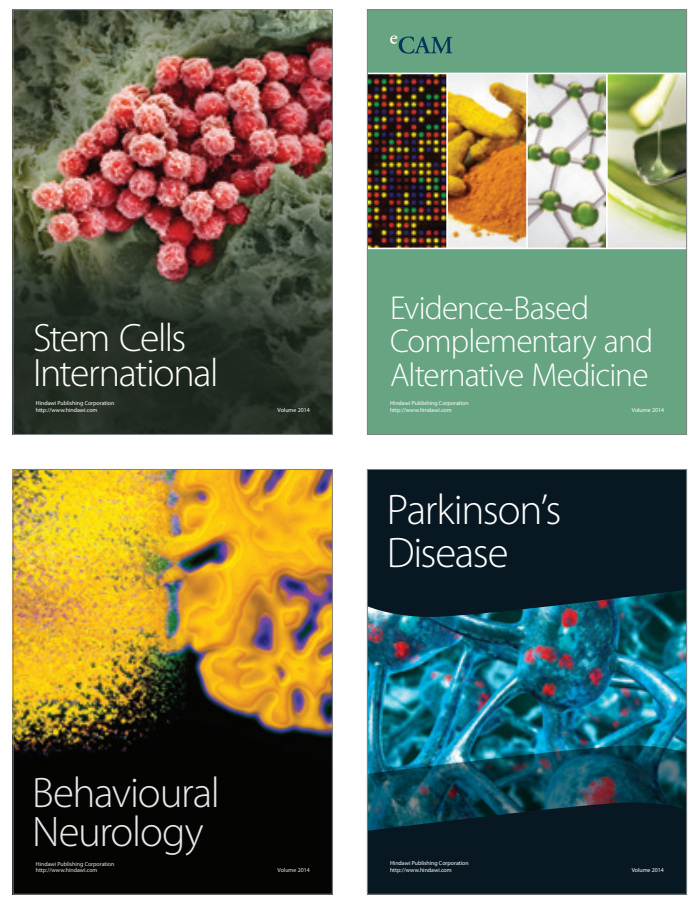
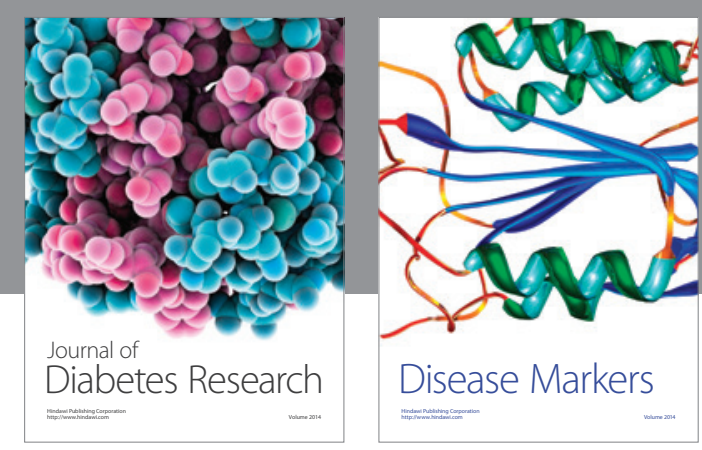

Disease Markers
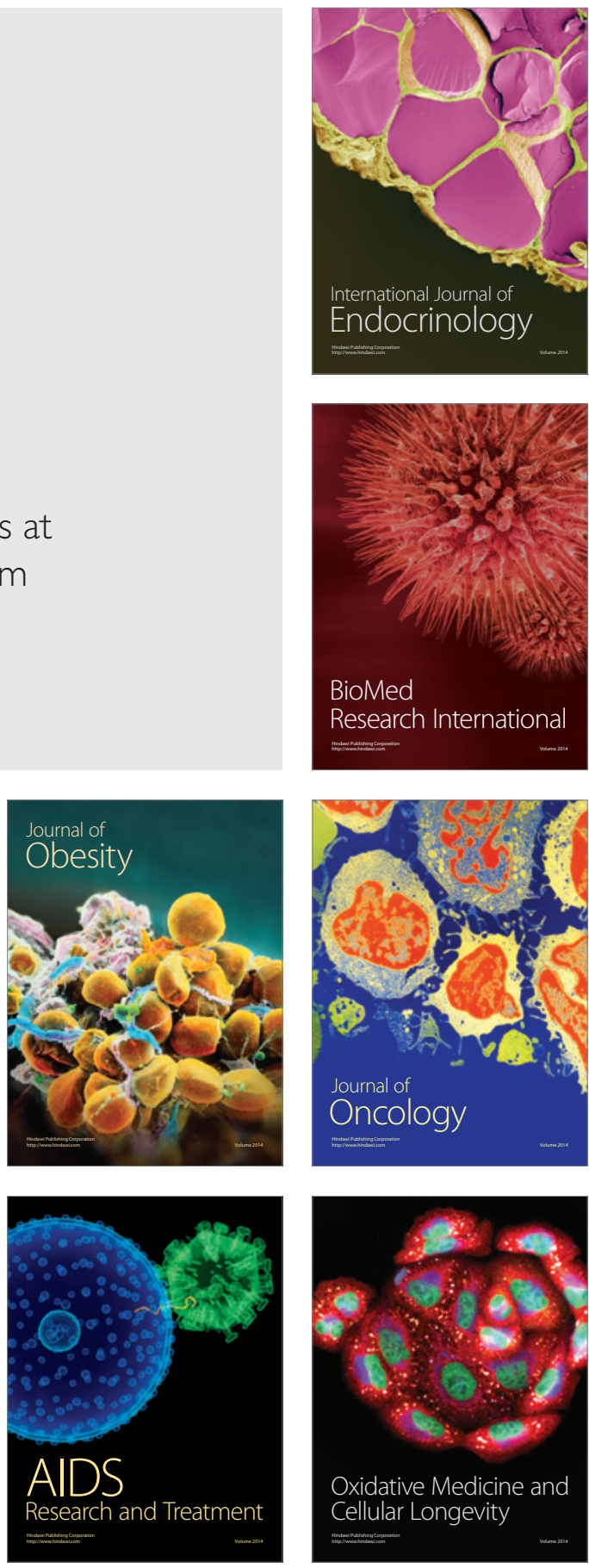